\title{
Experience of Mini-Percutaneous Nephrolithotomy in the Treatment of Large Impacted Proximal Ureteral Stones
}

\author{
Qilai Long Jianming Guo Zhibing Xu Yuanfeng Yang Hang Wang \\ Yanjun Zhu Yongkang Zhang Guomin Wang \\ Department of Urology, Zhongshan Hospital, Fudan University, Shanghai, China
}

\section{Key Words}

Mini-percutaneous nephrolithotomy · Ureter · Stones •

Proximal ureteral stones

\begin{abstract}
Objectives: To evaluate the efficacy and safety of minipercutaneous nephrolithotomy (PCNL) in the treatment of large impacted proximal ureteral stones. Methods: We retrospectively reviewed the outcomes of 163 patients who underwent mini-PCNL between January 2006 and August 2010. Mean age was 48.6 years and mean stone size was $18.4 \mathrm{~mm}$. Hydronephrosis and/or hydroureterosis appeared in all patients. In the prone position, percutaneous access (16-Fr sheath) was established by placement of an access needle into the intended calyx under fluoroscopic guidance or combined with ultrasound guidance for complete obstruction by stones while the contrast agent cannot transit. Pneumatic or ultrasonic probes were used throughout ureterorenoscopy for lithotripsy. The ureteral stents and nephrostomy tube were placed at the end of the procedure. Mean drop in hemoglobin, operative time, success rate, hospital stay, and complications were assessed. Results: MiniPCNL operations were performed successfully in all patients. Mean operation time was $37 \mathrm{~min}$. Mean postoperative hos-
\end{abstract}

pital stay was 3.6 days. All cases were followed up for $6-20$ months. No major complications like hemorrhage, perforation or organic injury were noted during the operation or postoperatively. The stone-free rate in all patients was $95.7 \%$. Calculus had no recurrence during the follow-up period. Hydronephrosis and hydroureterosis disappeared or were relieved. Conclusions: Mini-PCNL is a safe and effective therapy for large impacted proximal ureteral stones.

Copyright $\odot 2013$ S. Karger AG, Basel

\section{Introduction}

Technological advances have significantly improved the treatment of upper ureteral calculi. So far, various methods have been used including extracorporeal shockwavelithotripsy (ESWL), ureteroscopic lithotripsy (URL), percutaneous nephrolithotomy (PCNL), open surgery, and laparoscopic ureterolithotomy. However, it is still controversial which is the best for large, impacted proximal ureteral calculi.

ESWL was considered to be the primary therapy of uncomplicated upper urinary calculi for noninvasiveness, low morbidity and acceptable efficacy, but the stonefree rate was only $42.1-78.6 \%$ when the stone was $>1 \mathrm{~cm}$

\begin{tabular}{ll}
\hline KARGER & $\begin{array}{l}\text { ( ) 2013 S. Karger AG, Basel } \\
0042-1138 / 13 / 0904-0384 \$ 38.00 / 0 \quad \text { Karger }\end{array}$ \\
$\begin{array}{l}\text { E-Mail karger@karger.com } \\
\text { www.karger.com/uin }\end{array}$ & $\begin{array}{l}\text { This is an Open Access article licensed under the terms of the } \\
\text { Creative Commons Attribution-NonCommercial 3.0 Un- } \\
\text { ported license (CC BY-NC) (www.karger.com/OA-license), } \\
\text { applicable to the online version of the article only. Distribu- } \\
\text { tion permitted for non-commercial purposes only. }\end{array}$
\end{tabular}

Jianming Guo, MD, PhD

Department of Urology, Zhongshan Hospital

Fudan University, 180 Fenglin Road

Shanghai 200032 (China)

E-Mail guo.jianming@zs-hospital.sh.cn 
Table 1. Demographic and clinical characteristics of patients

$\begin{array}{lc}\text { Mean age (range), years } & 48.6(21-85) \\ \text { Male/female } & 101 / 62 \\ \text { Mean stone size (range), mm } & 18.4(12-30) \\ \text { Bilateral ureteral calculi } & 4(2.5 \%) \\ \text { Monolateral ureteral calculi } & 126(77.3 \%) \\ \text { Associated renal stones } & 33(20.2 \%) \\ \text { History of ESWL } & 17(10.3 \%) \\ \text { Previous open nephrolithotomy } & 19(11.6 \%)\end{array}$

[1-3]. The appearance of ureteroscopy has greatly improved the stone clearance rate, however the success rate of proximal ureteral stones has significantly declined compared with middle and lower ones $[4,5]$. Besides, proximal migration of the stone often leads to surgical failure. Laparoscopic or open surgical stone removal was only considered in rare cases when ESWL, URL, and PCNL failed or were unlikely to succeed. Therefore, a safe and effective method was urgently needed.

PCNL has been widely accepted as the treatment of choice for renal stones since the 1980s and became the gold-standard treatment for complex and large renal stones, but the indication of traditional PCNL was strictly limited due to its significant morbidity [6]. In order to reduce the complications and expand the use of PCNL, Helal et al. [7] developed a 'mini-perc' technique for treating pediatric calculi. Jackman et al. [8] then used a ureteroscopy sheath for PCNL to accomplish the 'mini-perc' technique. In China, Li et al. [9] also developed a modified mini-PCNL technique to manage most upper urinary tract stones. To evaluate the efficacy and safety of mini-PCNL in impacted proximal ureteral calculi since 2006, we started to use mini-PCNL (8-/9.8-Fr ureteroscopy via a 16-Fr percutaneous tract) for the treatment of impacted proximal ureteral calculi.

\section{Methods}

From January 2006 to August 2010, a total of 163 patients underwent antegrade mini-PCNL for upper ureteral calculi. The mean age of the patients was 48.6 years (range 21-85), and the male:female ratio was 101:62. The mean size of the calculus was $18.4 \mathrm{~mm}$ (range 12-30). The demographic and clinical characteristics of patients are shown in table 1 .

The inclusion criteria were: (a) stones located between the ureteropelvic junction and the upper border of the fourth lumbar vertebra, (b) upper ureteral stone $\geq 15 \mathrm{~mm}$ in largest diameter by plain film or ultrasound, and (c) with split glomerular filtration rate of the affected kidney $>15 \mathrm{ml} / \mathrm{min}$. In addition, patients with a stone diameter $>12 \mathrm{~mm}$ and a history of abdominal surgery, or repeated session of ESWL treatment, were also included to undergo the method. Exclusion criteria were uncorrected coagulopathy, pyonephrosis, or glomerular filtration rate $<15 \mathrm{ml} / \mathrm{min}$.

Preoperatively, patients were evaluated by a urine routine test, urine culture and drug sensitivity test, plain radiography of kidneys, ureters and bladder (KUB), and intravenous urography. Ultrasonography or unenhanced helical computed tomography for the degree of hydronephrosis, computed tomography urography (CTU) and radionuclide imaging were performed if necessary. Antibiotics were administered prophylactically to all patients with WBC-positive urine.

Calculus clearance was assessed on postoperative day 2 with a plain film of KUB. 'Stone-free' was defined as no residual stones or fragments $<4 \mathrm{~mm}$ detected on KUB, as fragments $<4 \mathrm{~mm}$ have a likelihood of passing spontaneously [10]. Bleeding during the operation was defined as bleeding which cannot be flushed out with sodium chloride within 2 min. Postoperative hemorrhage was defined as continued heavy bleeding which needed transfusion, embolism or nephrectomy. The operative time was calculated from performing the puncture to placing of the nephrostomy tube, which is also called skin-to-skin time. The time from insertion of the ureteric catheter to the turn in the prone position was not included.

The mini-PCNL procedure was performed under general anesthesia. It began with the placement of an open-ended 5-Fr ureteric catheter by cystoscopy with the patient in the lithotomy position. After the catheter was secured to a Foley catheter, the patient was placed in a prone position. Percutaneous access was obtained by the placement of an 18-gauge access needle into the intended calyx (most often the middle calyx, sometimes the upper calyx) under real-time X-ray guidance with the help of retrograde pyelography or combined with ultrasound guidance for complete obstruction by stones while the contrast agent cannot transit. It was commonly done through a middle posterior calyx. A 0.035 inch floppy-tipped guide wire was passed through the needle into the collecting system. The access needle was then removed and the skin and fascia were incised. Nephrostomy tract dilation was performed with serial dilators (Urovision) through the guide wire. The nephrostomy tract was dilated to 16 Fr to allow the passage of a 16-Fr peel-away sheath. A 8-/9.8-Fr Wolf rigid ureteroscope was used. Using a Swiss Lithoclast ${ }^{\circledR}$ for lithotripsy, most stone fragments $(<4 \mathrm{~mm})$ could be flushed out along with the backflow through the peel-away sheath, while the remaining big fragments were extracted with stone forceps. At the end of the procedure, a 5-Fr double-J stent and a 14-Fr nephrostomy tube was routinely placed to drain the kidney. Unless the plain film of KUB suggested significant residual calculi, the tube was removed $2-5$ days after the operation.

\section{Results}

All patients were treated with one session of percutaneous surgery. Among these, 159 patients underwent a single-tract mini-PCNL, 2 patients required a two-tract mini-PCNL for multiple kidney stones, and 2 patients underwent mini-PCNL on the contralateral side simulta- 
Table 2. Treatment results and complications

\begin{tabular}{ll}
\hline Variables & Results \\
\hline Mean operation time, min & $37(18-65)$ \\
Stone-free rate & $156 / 163(95.7 \%)$ \\
$\quad$ Single ureteral calculi & $128 / 130(98.5 \%)$ \\
Associated renal calculi & $28 / 33(84.8 \%)$ \\
Postoperative complications & $38(23.1 \%)$ \\
Fever & $24(14.6 \%)$ \\
Hematuria $>36$ h & $14(8.5 \%)$ \\
Perforation & 0 \\
Colonic or pleural injury & 0 \\
Postoperative hospital stay, days & $3.6(2-6)$ \\
\hline
\end{tabular}

neously. 156/163 (95.7\%) of the patients have complete calculus clearance. The stone-free rate in single ureteral calculi patients was $128 / 130$ (98.5\%), compared to $28 / 33$ $(84.8 \%)$ in patients associated with renal stones.

The average operating time was $37 \mathrm{~min}$ (range 18-65). 6 cases experienced bleeding during the process of renal penetration, all of whom completed the lithotripsy after pressure hemostasis. The mean hemoglobin drop was $1.52 \mathrm{~g} / \mathrm{dl}$ (range 0.6-3.9). 24 patients had transient fever $>38.5^{\circ} \mathrm{C}$ postoperatively. The mean postoperative hospital stay was 3.6 days (range 2-6). There were no significant complications such as hemorrhage necessitating transfusion, urinary tract perforation, or visceral injury that occurred during the procedure. The double-J stent was usually removed by cystoscope within 1 month after the operation. The results and complications are shown in table 2.

The mean follow-up time was 9 months (range 6-20); there no case of recurrence during the follow-up period. Hydronephrosis was to some degree relieved after the operation. Stone analysis revealed $73.2 \%$ of the calculi were mixed stones (the main component was calcium oxalate), $19.5 \%$ calcium oxalate, $3.7 \%$ carbonated apatite, $1.8 \%$ uric acid, and $1.8 \%$ cystine composition.

\section{Discussion}

Large upper ureteral calculi can cause complete obstruction resulting in hydroureteronephrosis, ultimately leading to renal function impairment. Therefore, timely effective treatment is the key to preventing renal injury.

Technical achievements have greatly changed the methods for the removal of ureteral stones, from open ureterolithotomy to ESWL, URL and PCNL, etc. Each device has its advantages and limitations, however the most optimal treatment for a large and impacted proximal ureteral stone remains controversial.

ESWL has proved to be safe and relatively effective for treating upper ureteral stones. Many centers state in their studies ESWL as first-line treatment for ureteral stones. Park et al. [2] have treated 218 patients with upper ureteral stones, achieving a $72.4 \%$ stone-free rate after a single ESWL session, but the rate decreases to $42 \%$ when the stone is $>1 \mathrm{~cm}$. High success rates of $>90 \%$ with ESWL have been previously reported, however it might be less effective (40-79\%) for large stones [1-3, 11, 12]. Besides, ESWL does not assure complete relief of obstruction and is often associated with prolonged attacks of pain during stone passage, flank soreness and repeated treatment in a substantial fraction of patients [13]. The reported retreatment rate of proximal ureteral calculi after ESWL can be up to $60 \%$ because large stones $(>10 \mathrm{~mm})$ and those causing a greater degree of hydronephrosis usually require more treatment sessions [14]. Therefore, we cannot consider ESWL as the primary choice when the size of ureteral stones is $>10 \mathrm{~mm}$.

Ureteroscopy with laser lithotripsy has been found to be more efficacious in the treatment of ureteral stones. Some urologists have recommended laser lithotripsy as first-line treatment $[15,16]$ and many articles reported that a stone-free rate for calculi $<10$ and $>10 \mathrm{~mm}$ was $90-100$ and $88-93 \%$, respectively [17-20]. Though a high stone clearance rate was attained, attention should be paid to the complications. The most common and serious complications are ureteral perforation. The reported perforation rates were $2-4.9 \%[21,22]$. Ureteral stricture is a late postoperative complication. Early reports [21] using 9.5- to 12.5-Fr ureteroscopes cited stricture rates of up to $4 \%$. Although flexible ureterorenoscopy with contact laser lithotripsy can be effective, a larger stone burden tackled by the retrograde route requires a longer procedure and increases the number of times the ureteroscope has to be reinserted into the ureter, increasing the chances of trauma, infection or endoscope damage. Many urologists therefore suggest that PCNL should be the primary therapy for large ureteral stones [23-25].

Although traditional PCNL has many advantages, such as clear vision, high stone clearance rate and short operation time, the indications were strictly limited for a number of serious complications [26]. Besides, the usual 26- to 34-Fr tract size of standard PCNL may be too large to be used in pediatric kidneys and in some adult undilated kidneys. Fortunately, some urologists have modi- 
fied the technique of standard PCNL by performing it with a miniature endoscope via a small percutaneous tract (11- to 20-Fr) and termed it as minimally invasive PCNL or mini-PCNL, making the treatment of upper ureteral stones with mini-PCNL a potential option. Many authors state the 'mini-perc' technique is believed to have several superiorities including decreased blood loss, increased maneuverability, and shorter hospital stay $[8,27]$. Zhu et al. [23] studied 192 patients with proximal ureteral calculi and severe hydronephrosis who underwent PCNL with a different lithotriptor; the overall stone-free rate was $86.5 \%$ (range $78.9-92.9 \%$ ) and most complications were minor and insignificant except for 3 patients who needed blood transfusion for severe bleeding. Juan et al. [24] compared PCNL and URL for large impacted upper ureteral calculi $>15 \mathrm{~mm}$; the stone-free rate at the 1-month follow-up was $95.4 \%$ in the PCNL group, while it was $58 \%$ the URL group ( $<<0.001)$. Two patients in the PCNL group had blood loss requiring transfusion and 8 patients had stones showing upward migration during the URL procedure, which suggested that for an impacted, proximal ureteral stone $>15 \mathrm{~mm}$ in diameter, PCNL had better stone-free rates and could simultaneously treat coexisting renal stones. Goel et al. [25] reported 66 patients who underwent PCNL for impacted proximal ureteral calculi with a result of 65 (98.5\%) patients who had complete calculus clearance in a single session through a single tract. Mean operating time and hospital stay were $47 \mathrm{~min}$ and $46 \mathrm{~h}$, respectively, and there were no significant postoperative complications. Considering the cost of each method, they believed that an antegrade percutaneous approach can be a reasonable option in developing countries.

The main aim of stone operation is to get a high stone clearance rate, so it is important to deal with the stone fragments effectively. Clinically insignificant residual fragments (CIRFs) after PCNL remains a major concern, e.g. Skolarikos and Papatsoris [28] believed that if CIRFs were left untreated, approximately half of the patients would experience a stone-related event for which more than a half would also need a secondary surgical intervention. Altunrende et al. [29] investigated 38 patients with CIRFs after PCNL and found that $47.4 \%$ had a symptomatic episode that necessitated medical therapy or an increase in the size of the fragments with a median follow-up of $28.4 \pm 5.3$ months. In order to effectively deal with the stone fragments, during the procedure we used the peel-away sheath to encompass the stone. Under direct vision, the stone was fragmented by pneumatic lithotripsy and most stone fragments $(<4 \mathrm{~mm})$ would flush out along with the backflow through the peel-away sheath; the remaining big fragments were extracted with stone forceps. Together with a 5-Fr ureteral catheter which can prevent the stone fragments from dropping into the distal ureter, we can effectively flush the fragments out and assure a high stone clearance rate.

In our center, we use 8-/9.8-Fr ureteroscopy via a small percutaneous tract (16-Fr) to treat impacted upper ureteral calculi, which has resulted in being a safe and effective procedure. In 163 patients, we achieved a complete stone clearance rate of $95.7 \%$ after one procedure. The mean postoperative hospital stay is 3.6 days (range 2-6). Except for bleeding during the operation in 6 patients, 24 had transient fever $>38.5^{\circ} \mathrm{C}$, and 14 patients had hematuria $>36$ h postoperatively. We did not experience any major complications such as hemorrhage necessitating transfusion, urinary leakage, visceral injuries or sepsis. Our experiences are the following: (a) A thorough preoperative examination can effectively reduce the chance of sepsis. (b) Percutaneous renal access was carried out by a skillful surgeon; when there is difficulty with the puncture, combined ultrasound guidance and fluoroscopic guidance may be useful. (c) Skilled teamwork is greatly helpful in shortening the operative time, which can in turn reduce the complications of a longer operation time associated with both septic shock and severe renal bleeding [30]. In our center, more and more patients are expected to undergo a mini-PCNL which can achieve a complete stone clearance in a single step. Mini-PCNL has therefore become a preferred choice. In addition, patients with ureteric strictures, tortuous ureter, ileal conduit and proximal ureteric stones are appropriate candidates for PCNL and might benefit from mini-PCNL.

\section{Conclusions}

Mini-PCNL greatly reduces the complications of PCNL. Though the primary treatment of impacted proximal ureteral calculi is still controversial, mini-PCNL provides another option for urologists. Sometimes, with skillful experience, it might be an advantageous choice.

\section{Disclosure Statement}

The authors have no conflicts of interest to disclose. 


\section{References}

1 Ziaee SA, Halimiasl P, Aminsharifi A, et al: Management of 10 - to $15-\mathrm{mm}$ proximal ureteral stones: ureteroscopy or extracorporeal shockwave lithotripsy? Urology 2008;71: 28-31.

-2 Park H, Park M, Park T: Two-year experience with ureteral stones: extracorporeal shockwave lithotripsy vs. ureteroscopic manipulation. J Endourol 1998;12:501-504

-3 Kato Y, Yamaguchi S, Hori J, Okuyama M, Kakizaki H: Improvement of stone comminution by slow delivery rate of shock waves in extracorporeal lithotripsy. Int J Urol 2006; 13:1461-1465.

4 Kassem A, Elfayoumy H, Elsaied W, Elgammal M, Bedair A: Laser and pneumatic lithotripsy in the endoscopic management of large ureteric stones: a comparative study. Urol Int 2012;88:311-315.

-5 Isen K: Pneumatic ureteroscopic lithotripsy: is it still a reasonable treatment option for multiple ureteric stones? Urol Int 2012;88: 316-321.

-6 Wickham JE, Kellet MJ: Percutaneous nephrolithotomy. Br Med J Clin Res Ed 1981;283: 1571-1572.

$>7$ Helal M, Black T, Lockhart J, Figueroa TE: The Hickman peel-away sheath: alternative for pediatric percutaneous nephrolithotomy. J Endourol 1997;11:171-172.

$>8$ Jackman SV, Docimo SG, Cadeddu JA, et al: The 'mini-perc' technique: a less invasive alternative to percutaneous nephrolithotomy. World J Urol 1998;16:371-374.

$>9 \mathrm{Li}$ X, He Z, Wu K, et al: Chinese minimally invasive percutaneous nephrolithotomy: the Guangzhou experience. J Endourol 2009;23: 1693-1697.

$>10$ Silverstein AD, Terranova SA, Auge BK, et al: Bilateral renal calculi: assessment of staged vs. synchronous percutaneous nephrolithotomy. J Endourol 2004;18:145-151.
11 Tiselius HG: How efficient is extracorporeal shockwave lithotripsy with modern lithotripters for removal of ureteral stones? J Endourol 2008;22:249-255.

12 White W, Klein F: Five-year clinical experience with the Dornier Delta lithotriptor. Urology 2006;68:28-32.

13 Tawfick ER: Treatment of large proximal ureteral stones: extracorporeal shock wave lithotripsy versus semi-rigid ureteroscope with lithoclast. Int Arch Med 2010;3:3.

14 Singh I, Gupta NP, Hemal AK, et al: Impact of power index, hydroureteronephrosis, stone size, and composition on the efficacy of in situ boosted ESWL for primary proximal ureteral calculi. Urology 2001;58: 16-22.

15 Lee YH, Tsai JY, Jiaan BP, Wu T, Yu CC: Prospective randomized trial comparing shockwave lithotripsy and ureteroscopic lithotripsy for management of large upper third ureteral stones. Urology 2006;67:480-484.

16 Fong YK, Ho SH, Peh OH, et al: Extracorporeal shockwave lithotripsy and intracorporeal lithotripsy for proximal ureteric calculi - a comparative assessment of efficacy and safety. Ann Acad Med Singapore 2004; 33:80-83.

17 Parker BD, Frederick RW, Reilly TP, Lowry PS, Bird ET: Efficiency and cost of treating proximal ureteral stones: shockwave lithotripsy versus ureteroscopy plus holmium: yttrium-aluminum-garnet laser. Urology 2004;64:1102-1106.

18 Lam JS, Greene TD, Gupta M: Treatment of proximal ureteral calculi: holmium:YAG laser ureterolithotripsy versus extracorporeal shock wave lithotripsy. J Urol 2002;167: 1972-1976.

19 Youssef RF, El-Nahas AR, El-Assmy AM, et al: Shockwave lithotripsy versus semirigid ureteroscopy for proximal ureteral calculi $(<20 \mathrm{~mm})$ : a comparative matched-pair study. Urology 2009;73:1184-1187.

$>20$ Muslumanoglu AY, Karadag MA, Tefekli $\mathrm{AH}$, et al: When is open ureterolithotomy indicated for the treatment of ureteral stones? Int J Urol 2006;13:1385-1388.
21 Johnson DB, Pearle MS: Complications of ureteroscopy. Urol Clin North Am 2004;31: 157-171.

22 Ceylan K, Sunbul O, Sahin A, Gunes M: Ureteroscopic treatment of ureteral lithiasis with pneumatic lithotripsy: analysis of 287 procedures in a public hospital. Urol Res 2005;33:422-425.

23 Zhu Z, Xi Q, Wang S, et al: Percutaneous nephrolithotomy for proximal ureteral calculi with severe hydronephrosis: assessment of different lithotriptors. J Endourol 2010; 24:201-205.

24 Juan YS, Shen JT, Li CC, et al: Comparison of percutaneous nephrolithotomy and ureteroscopic lithotripsy in the management of impacted, large, proximal ureteral stones. Kaohsiung J Med Sci 2008;24:204-208.

25 Goel R, Aron M, Kesarwani PK, et al: Percutaneous antegrade removal of impacted upper-ureteral calculi: still the treatment of choice in developing countries. J Endourol 2005;19:54-57.

26 Amer T, Ahmed K, Bultitude M, et al: Standard versus tubeless percutaneous nephrolithotomy: a systematic review. Urol Int 2012; 88:373-382.

27 El-Nahas AR, Shokeir AA, El-Kenawy MR, et al: Safety and efficacy of supracostal percutaneous nephrolithotomy in pediatric patients. J Urol 2008;180:676-680.

28 Skolarikos A, Papatsoris AG: Diagnosis and management of postpercutaneous nephrolithotomy residual stone fragments. J Endourol 2009;23:1751-1755.

29 Altunrende F, Tefekli A, Stein RJ, et al: Clinically insignificant residual fragments after percutaneous nephrolithotomy: mediumterm follow-up. J Endourol 2011;25:941-945.

30 Wang Y, Jiang F, Hou Y, et al: Post-percutaneous nephrolithotomy septic shock and severe hemorrhage: a study of risk factors. Urol Int 2012;88:307-310. 